\title{
Performance of LI-RADS version 2018 CT treatment response algorithm in tumor response evaluation and survival prediction of patients with single hepatocellular carcinoma after radiofrequency ablation
}

\author{
Yun Zhang ${ }^{1 \#}$, Jinju Wang ${ }^{2 \#}$, Hui $\mathrm{Li}^{2}$, Tianying Zheng ${ }^{1}$, Hanyu Jiang ${ }^{1}$, Mou Li ${ }^{1}$, Bin Song ${ }^{1}$ \\ ${ }^{1}$ Department of Radiology, Sichuan University West China Hospital, Chengdu 610041, China; ${ }^{2}$ Department of Liver Surgery \& Liver \\ Transplantation Center, West China Hospital, Chengdu 610041, China \\ Contributions: (I) Conception and design: B Song, Y Zhang; (II) Administrative support: B Song; (III) Provision of study materials or patients: J Wang, \\ H Li; (IV) Collection and assembly of data: Y Zhang, T Zheng; (V) Data analysis and interpretation: H Jiang, M Li; (VI) Manuscript writing: All \\ authors; (VII) Final approval of manuscript: All authors. \\ \#These authors contributed equally to this work. \\ Correspondence to: Bin Song, MD, Professor. Department of Radiology, Sichuan University West China Hospital, No. 37 Guoxue Alley, Chengdu \\ 610041, China. Email: songlab_radiology@163.com.
}

Background: The Liver Imaging Reporting and Data System treatment response algorithm (LI-RADS TRA) was developed to evaluate the tumor response of patients with hepatocellular carcinoma (HCC) after locoregional treatments. This study aimed to evaluate the performance of LI-RADS computed tomography (CT) TRA version 2018 in tumor response assessment and survival prediction of patients with single HCC after radiofrequency ablation (RFA).

Methods: Forty patients who underwent RFA for single HCC between 2010 and 2016 were included in this retrospective study. The overall survival (OS) data from all the patients after the first therapy was collected. Two readers independently assessed the pretreatment (within $7 \mathrm{~d}$ ) and posttreatment (within $90 \mathrm{~d}$ after RFA) CT manifestations using the LI-RADS version 2018 CT TRA. Inter-reader agreement was assessed. Another radiologist re-evaluated any divergent results and came to the final conclusion. The performance of LI-RADS version 2018 CT TRA for tumor response assessment and predicting survival of patients with single HCC after RFA was evaluated.

Results: Interobserver agreement was moderate between the 2 readers [ $\kappa=0.602,95 \%$ confidence interval (CI): 0.390-0.814] when using LI-RADS version 2018 TRA to evaluate tumor response for patients with single HCC after RFA. Patients classified as LR-TR viable had significantly lower OS than those classified as LR-TR nonviable $(\mathrm{P}=0.005)$ and LR-TR equivocal $(\mathrm{P}=0.036)$. However, the OS between LR-TR nonviable and LR-TR equivocal did not differ significantly $(\mathrm{P}=0.901)$.

Conclusions: LI-RADS version 2018 CT TRA can be applied to predict viable or nonviable HCC after RFA. Patients with LR-TR viable had significantly lower OS than those with LR-TR nonviable and LRTR equivocal. More research is needed to validate the performance of LI-RADS version 2018 TRA in HCC tumor response evaluation, to better grasp the use of the tie-breaking rule, and to improve the accuracy of prediction for tumor viability.

Keywords: Hepatocellular carcinoma (HCC); radiofrequency ablation (RFA); Liver Imaging Reporting and Data System (LI-RADS); treatment response algorithm (TRA); computed tomography (CT)

Submitted Feb 20, 2020. Accepted for publication Mar 02, 2020.

doi: 10.21037/atm.2020.03.120

View this article at: http://dx.doi.org/10.21037/atm.2020.03.120 


\section{Introduction}

Hepatocellular carcinoma (HCC) accounts for 75-80\% of liver cancer, and has the third highest cancer-related mortality rate (1). Liver-directed locoregional therapies, including transcatheter and image-guided treatment, such as radiofrequency ablation (RFA), transarterial chemoembolization (TACE), and combined therapy, have been widely accepted in Western countries as a bridging or downstage treatment for patients with HCC who are awaiting liver transplantation (2). In China, however, the lack of liver-related resources and the high cost of surgery have made locoregional therapies to be more frequently used as an attempt to cure HCC. The timely and reliable assessment of tumor response after locoregional therapy is essential for guiding clinical decision-making and the implementation of personalized treatment for HCC.

There have been several guidelines and criteria developed by different organizations for assessing tumor response of patients with HCC in recent decades. According to the evaluation basis, these standards can be divided into 2 categories: (I) those based on tumor load, including the World Health organization (WHO) criteria (3) and the Response Evaluation Criteria in Solid Tumors (RECIST) criteria $(4,5)$; and (II) those based on tumor activity, such as the European Association for the Study of the Liver (EASL) criteria (6), Choi criteria (7), and the modified RECIST (mRECIST) criteria (8). Previous studies have proven that guidelines or criteria based on tumor activity are more informative in evaluating tumor response of locoregional treatment for HCC (9-12).

The Liver Imaging Reporting and Data System (LIRADS) has been updated and optimized in the past few years and is used for standardizing the collection, interpretation, reporting, and evaluation of data for patients at risk of developing HCC (13-15). In 2017, LI-RADS proposed the treatment response algorithm (TRA), which was better explained and standardized in the 2018 version and aimed at assisting radiologists to better interpret computed tomography/magnetic resonance imaging (CT/ MRI) of HCC after locoregional treatment. Unlike the existing evaluation criteria described above, whose criteria only focus on disease progression, Liver Imaging Reporting and Data System treatment response algorithm (LI-RADS TRA) captures the tumor necrosis of each lesion. A treated lesion with nodular, mass-like, or thick irregular tissue in or alongside it was recommended as the most significant feature for defining a viable tumor by LI-RADS TRA.
Moreover, arterial phase hyperenhancement (APHE), washout appearance, or similar characteristics of enhancement to pretreatment are 3 other ancillary imaging features that help to establish the diagnosis of a viable tumor. In addition, the treatment-specific, expected enhancement patterns are clearly explained in LI-RADS TRA to facilitate radiological identification of pseudoprogression after treatment.

Although there have been several studies using pathology as the gold standard to investigate the value of LI-RADS TRA in the assessment of tumor response of HCC after locoregional treatment (16-20); to our knowledge, the performance of LI-RADS 2018 TRA in tumor response evaluation and the survival prediction of patients with HCC after locoregional therapy have not been reported.

In this study, we aimed to evaluate the performance of LI-RADS version 2018 CT TRA in tumor response assessment and survival prediction of patients with single HCC after RFA. In addition, we evaluated the inter-reader agreement for defining viable, nonviable, and equivocal tumors on CT images.

\section{Methods}

\section{Patients}

This study was approved by our institutional review board. The requirement for patient consent was waived because of the study's retrospective design. From January 2010 to December 2016, 470 adult patients underwent RFA for HCC at our institution's Department of Liver Surgery \& Liver Transplantation Center. Two surgeons collected all the patients' clinical, laboratory, and survival data from the electronic medical record system. One radiologist with 5 years' experience in abdominal imaging who was not involved in this study reviewed the electronic medical records and picture archiving and communication system (PACS) records to verify the information of all included patients.

All patients were confirmed by imaging, pathology, or needle biopsy. The inclusion criteria were as follows: (I) single HCC (lesion diameter $>5 \mathrm{~mm}$ ); (II) patients who underwent RFA; (III) good liver function (i.e., Child-Pugh A or B), without vascular tumor thrombus or adjacent organs invasion; (IV) patients who underwent dynamic enhanced liver CT pre- (within $7 \mathrm{~d}$ ) or after RFA (within $90 \mathrm{~d}$ ); and $(\mathrm{V})$ clinical and follow-up results. Of 470 patients, 430 were excluded because of the following criteria: (I) nonsingle HCC ( $n=77)$; (II) previously treated (i.e., surgical 


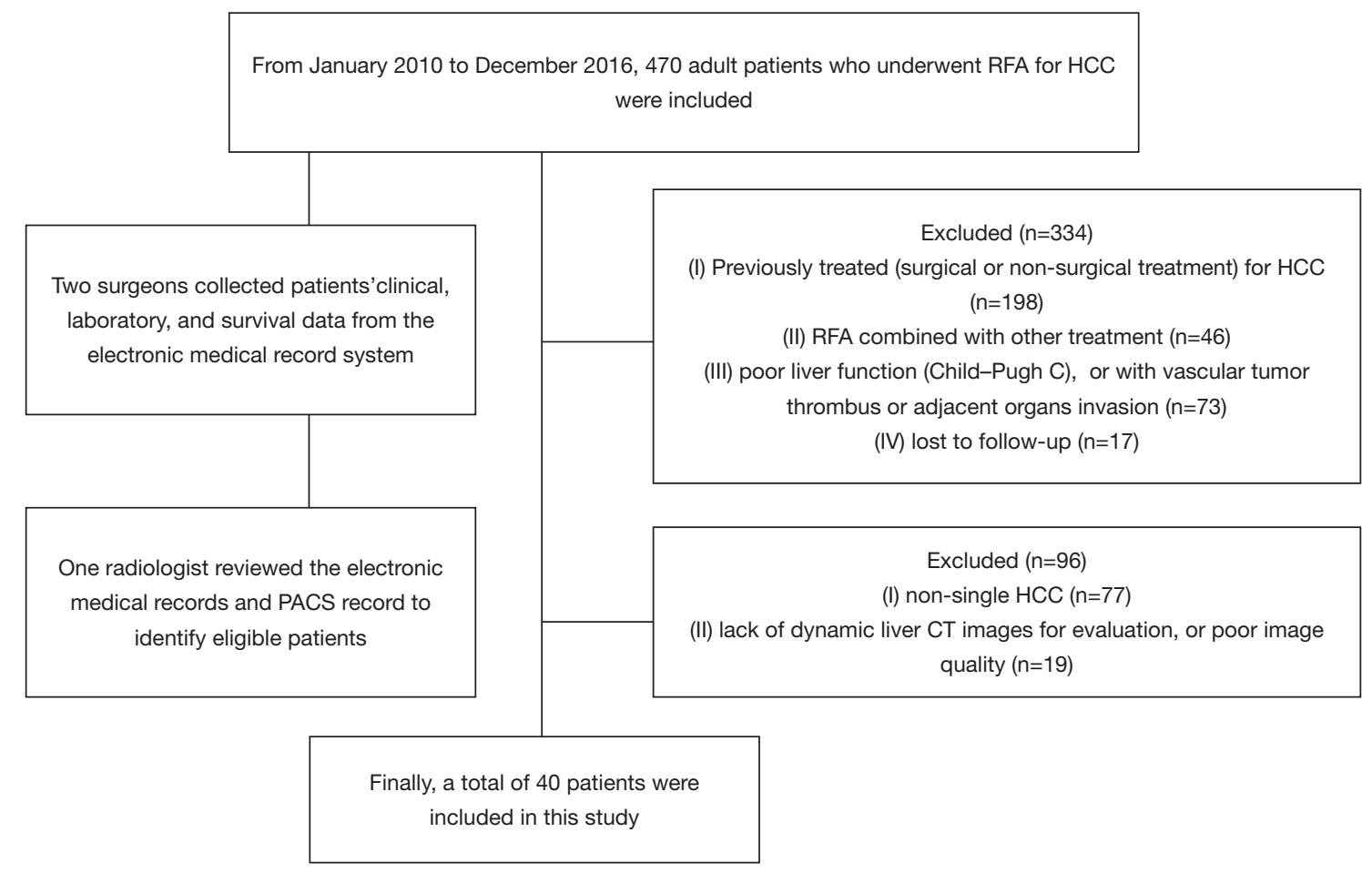

Figure 1 Flow diagram of the patients selection. RFA, radiofrequency ablation; HCC, hepatocellular carcinoma.

or non-surgical treatment) for HCC $(n=198)$; (III) RFA combined with other treatment $(n=46)$; (IV) poor liver function (i.e., Child-Pugh C), or with vascular tumor thrombus, or adjacent organs invasion $(n=73)$; (V) lack of dynamic liver CT images for evaluation, or poor image quality $(n=19)$; and (VI) lost to follow-up $(n=17)$. Finally, 40 patients were included (Figure 1).

\section{RFA techniques}

All 40 patients underwent at least 1 RFA, including percutaneous, laparoscopic, or laparotomic ablations. The operation area was disinfected and sterilized. Local infiltration anesthesia at the puncture point or general anesthesia was applied. The type of ablation needle was selected according to the lesion size, and then ablation was conducted for 5-12 min. Ultrasound or CT scan was performed again when ablation was completed to confirm that there was no active bleeding or adjacent organ damage.

\section{Imaging techniques}

In this study, we used a 64-row multidetector (MD) CT scanner (Brilliance 64; Philips Medical Systems, Eindhoven,
Netherlands), a 128-row MDCT (Somatom Definition AS+; Siemens Healthcare Sector, Forchheim, Germany), and a dual-source CT system (Somatom Definition Flash; Siemens Healthcare Sector, Forchheim, Germany) to scan patients using the following parameters: $120 \mathrm{kV}$ (voltage), 200-210 mA (current), $0.5-0.75 \mathrm{~s}$ (rotation time), $0.625 \mathrm{~mm}$ (detector collimation), 0.8-1.0 (pitch), 2.1-5.0 mm (section thickness). Omnipaque (350; GE Healthcare), a contrast medium, was intravenously injected at a rate of $3 \mathrm{~mL} / \mathrm{s}$. The trigger threshold of the aorta reached $100 \mathrm{HU}$, and the abdominal arterial phase was performed $35 \mathrm{~s}$ after the trigger. The portal phase was performed $70 \mathrm{~s}$ after the trigger.

\section{Image analysis}

The radiologist who did not participate in this study marked the images corresponding to the scanning date so that 2 reviewers could select the eligible images to assess. Two double-blinded abdominal radiologists with 7 and 8 years' experience in liver imaging reviewed the CT images. Both reviewers were informed that all the patients had undergone RFA for HCC. However, they were not informed about the clinical, laboratory, and survival results. 
Several key imaging features according to LI-RADS 2018 TRA were recorded by the 2 reviewers, including (I) enhancing nodular, or irregular tissue in or along the margin of the treated lesion; (II) APHE; (III) washout; and (IV) treatment-specific enhancement patterns of RFA. The tumor response categories were as follows: (I) LR-TR viable, (II) LR-TR nonviable, and (III) LR-TR equivocal. Another radiologist with $>15$ years' experience of abdominal imaging re-evaluated the divergent results and came to the final conclusion.

\section{Follow-up}

To verify the evaluation of LI-RADS 2018 CT TRA in assessment of tumor response and survival prediction of patients with single HCC, we evaluated the images of the first CT examination within 90 days after treatment as the posttreatment image. The end of the follow-up period was December 1, 2019. The overall survival (OS) of all patients was recorded during follow-up.

\section{Statistical analysis}

Statistical analyses were performed using SPSS version 23.0 (SPSS Inc., Chicago, IL, USA). Categorical variables are shown as counts and percentages. The kappa test was used to assess the interobserver agreement for LI-RADS 2018 CT TRA of the treated lesion after RFA. The criteria for assessment were as follows (21): $\kappa=0$, no agreement; $0<\kappa<0.20$, slight agreement; $\kappa=0.21-0.40$, fair agreement; $\kappa=0.41-0.60$, moderate agreement; $\kappa=0.61-0.80$, good agreement; and $\kappa=0.81-1.0$, excellent agreement. $\mathrm{P}<0.05$ was considered to be a statistically significant value. In addition, we used Kaplan-Meier statistics to draw survival curves, and the log-rank test was used to compare differences between curves. By analyzing the relationship between radiological and survival results, we validated the value of LI-RADS 2018 CT TRA for tumor response evaluation and survival prediction of patients with single HCC after RFA.

\section{Results}

\section{Clinical characteristics of patients}

The demographics of the 40 patients are shown in Table 1 . Of all the 40 patients, 35 patients $(87.5 \%)$ were male. The mean age of included patients was $60.32 \pm 10.4$ (ranging from 43 to 78 years old). Hepatitis virus infection was the most common etiology and included hepatitis B $(32 ; 80 \%)$ and hepatitis C $(2 ; 5.0 \%)$. Thirty-two $(80 \%)$ patients underwent only one RFA treatment, and eight (20\%) patients underwent more than one RFA treatment.

\section{Baseline pathological and imaging features}

All the patients underwent routine tests for liver function, and the baseline laboratory examination results were as follows: average $\alpha$-fetoprotein $=217.90(1.3-1876) \mathrm{ng} / \mathrm{mL}$; average carbohydrate antigen-199 $=58.20(1.17-180.5) \mathrm{U} / \mathrm{mL}$; average serum aspartate aminotransaminase $\geq 35 \mathrm{IU} / \mathrm{L}$ $=18$ cases $(45 \%)$; average serum alanine aminotransferase $\geq 40 \mathrm{IU} / \mathrm{L}=14$ cases $(34 \%)$; and average total bilirubin $\geq 21 \mu \mathrm{mol} / \mathrm{L}=12$ cases $(30 \%)$. Moreover, all 40 patients underwent dynamic enhanced abdominal CT examination. In the analysis of the baseline CT imaging, it was found that $72.5 \%$ of HCCs were located in the right half of the liver. The largest diameter of $60 \%$ of HCC was $<3 \mathrm{~cm}$.

\section{Interobserver agreement}

The 2 radiologists with 7 and 8 years' experience of liver imaging independently assessed the pretreatment (within $7 \mathrm{~d}$ ) and posttreatment (within $90 \mathrm{~d}$ ) CT manifestations by the LI-RADS 2018 CT TRA. Inter-reader agreement was assessed by using the kappa test. Interobserver agreement between the 2 readers using LI-RADS 2018 TRA was moderate and close to good $[\kappa=0.602,95 \%$ confidence interval (CI): 0.390-0.814] (Table 2). Another radiologist (R3) with $>15$ years' experience of abdominal imaging re-evaluated the divergent results and came to the final conclusion (Table 3).

\section{Performance of LI-RADS 2018 CT TRA in tumor response and survival prediction}

All 40 patients were evaluated and classified into 3 categories according to LI-RADS 2018 CT TRA. The patients were classified according to CT features as follows: (I) LR-TR viable: a treated lesion with enhancing nodular or mass-like, or thick irregular tissue in or along the margin, with 1 of the following: APHE, washout, and enhancement pattens similar to pretreatment (Figure 2); (II) LR-TR nonviable: no enhancement in the treated lesion, or with specific-expected enhancement pattern (Figure 3); (III) LRTR equivocal: insufficient evidence to support a conclusion for LR-TR viable or LR-TR nonviable (Figure 4). 
Table 1 Patient demographics and baseline characteristics

\begin{tabular}{|c|c|}
\hline Variable & Number (\%) \\
\hline \multicolumn{2}{|c|}{ Clinical characteristics (No. of patients $=40$ ) } \\
\hline Age (years) & $60.32 \pm 10.4$ (range, $43-78)$ \\
\hline Male/female (gender) & $35 / 5$ (male $87.5 \%$ ) \\
\hline \multicolumn{2}{|l|}{ Etiology of liver disease } \\
\hline HBV & $32(80 \%)$ \\
\hline $\mathrm{HCV}$ & $2(5.0 \%)$ \\
\hline \multicolumn{2}{|c|}{ Total number RFA treatment } \\
\hline 1 & $32(80 \%)$ \\
\hline 2 & $3(7.5 \%)$ \\
\hline$>5$ & $5(12.5 \%)$ \\
\hline \multicolumn{2}{|c|}{ Baseline laboratory examination } \\
\hline AFP level (ng/mL) & 217.90 (range, $1.3-1,876$ ) \\
\hline CA-199 (U/ml) & 58.20 (range, 1.17-180.5) \\
\hline Serum AST $(\geq 35 \mathrm{IU} / \mathrm{L})$ & $18(45 \%)$ \\
\hline Serum ALT ( $\geq 40$ IU/L) & $14(34 \%)$ \\
\hline Tbil $(\geq 21 \mu \mathrm{mol} / \mathrm{L})$ & $12(30 \%)$ \\
\hline \multicolumn{2}{|c|}{ Baseline imaging features of $\mathrm{HCC}$} \\
\hline \multicolumn{2}{|l|}{ Tumor location } \\
\hline Left-half liver & $9(22.5 \%)$ \\
\hline Right-half liver & $29(72.5 \%)$ \\
\hline The caudate lobe & $2(5 \%)$ \\
\hline \multicolumn{2}{|c|}{ The maximum diameter of lesion $(\mathrm{cm})$} \\
\hline $1<\mathrm{d}<2$ & $9(22.5 \%)$ \\
\hline $2 \leq \mathrm{d}<3$ & $15(37.5)$ \\
\hline$d>3$ & $16(40 \%)$ \\
\hline
\end{tabular}

(Data in parentheses are percentages). HAV, hepatitis A virus infection; HBV, hepatitis B virus infection; HCV, hepatitis C virus infection; RFA, radiofrequency ablation; AFP, alphafetoprotein; CA-199, carbohydrate antigen-199; AST, aspartate aminotransaminase; Tbil, total bilirubin; HCC, hepatocellular carcinoma.

OS of all patients was recorded until December 1, 2019. The survival curve of OS was drawn by KaplanMeier statistics, and log-rank test was used to compare differences between curves (Figure 5). Patients classified as LR-TR viable had significantly lower OS than patients classified as LR-TR nonviable $(\mathrm{P}=0.005)$ and LR-TR equivocal $(\mathrm{P}=0.036)$. Moreover, patients classified as LR-
TR nonviable appeared to have higher OS than those classified as LR-TR nonviable in the 50 months after RFA. From month 51, the OS of the latter was higher than that of the former. The log-rank test showed that there was no significant difference $(\mathrm{P}=0.901)$.

\section{Discussion}

This study demonstrated that LI-RADS version 2018 TRA can be applied to predict viable or nonviable HCC after RFA. Furthermore, patients classified as LR-TR viable had significantly lower OS than those classified as LR-TR nonviable and LR-TR equivocal. However, the OS between LR-TR nonviable and LR-TR equivocal patients did not differ significantly.

The purpose of LI-RADS is to standardize the collection, interpretation, reporting, and evaluation of patients at risk of HCC. Previous studies have demonstrated that use of LI-RADS improved the diagnostic performance of HCC and reclassified high-risk liver nodules with the help of ancillary features on imaging (22-24). It was also found that, compared with other noninvasive imaging diagnostic methods, LI-RADS shows better sensitivity and accuracy in LR-5 category diagnosis and characterization, which has mostly been attributed to the application of diffusion-weighted imaging (25) and hepatobiliary phase scanning (26). The primary purpose of developing LIRADS TRA is to improve the interobserver agreement in the interpretation of imaging features on CT/MRI so as to provide clinicians with more reliable tumor response evaluation and curative efficacy prediction.

Several pilot studies used histopathology as the gold standard to investigate the performance of LI-RADS TRA in predicting viable or nonviable tumors after treatment. Chaudhry (16) et al. showed good ( $\kappa=0.71)$ interobserver agreement using LI-RADS MRI TRA for predicting tumor viability of HCC treated with RFA. In our study, the interobserver agreement was moderate $(\kappa=0.602)$. These discrepancies might be attributable to the different techniques used in the 2 studies and the differences in diagnostic levels between observers. Cools (17) et al. validated the performance of LI-RADS TRA in evaluating tumor viability of small HCC $(<3 \mathrm{~cm})$ after thermal ablation. The results showed that LR-TRA was highly sensitive but less accurate, because many pathologically proven LR-TR viable cases were mistakenly classified as LR-TR equivocal. The same conclusion was reached by Chaudhry (16) et al. and Shropshire (19) et al. 
Table 2 Interobserver agreement between the two readers

\begin{tabular}{lllll}
\hline \multirow{2}{*}{ Reader 2} & \multicolumn{4}{c}{ Reader 1} \\
\cline { 2 - 5 } & $\begin{array}{l}\text { LR-TR } \\
\text { nonviable }\end{array}$ & $\begin{array}{l}\text { LR-TR } \\
\text { equivocal }\end{array}$ & $\begin{array}{l}\text { LR-TR } \\
\text { viable }\end{array}$ & Total \\
\hline LR-TR nonviable & $16(40 \%)$ & $5(12.5 \%)$ & $0(0 \%)$ & $21(52.5 \%)$ \\
LR-TR equivocal & $3(7.5 \%)$ & $6(15 \%)$ & $0(0 \%)$ & $9(22.5 \%)$ \\
LR-TR viable & $0(0 \%)$ & $2(5 \%)$ & $8(20 \%)$ & $10(22.5 \%)$ \\
Total & $19(47.5 \%)$ & $13(32.5 \%)$ & $8(20 \%)$ & $40(100 \%)$ \\
\hline
\end{tabular}

Data in parentheses are percentages. Percentages may total $100 \%$ because of rounding. LI-RADS, Liver Imaging Reporting and Data System; TRA, tumor response algorithm; LR-TR, Liver Imaging Reporting and Data System Tumor Response.

Table 3 Final conclusion (reevaluated by Reader 3) by using LIRADS 2018 TRA

\begin{tabular}{ll}
\hline LI-RADS TRA category & No. patients \\
\hline LR-TR nonviable & $24(60 \%)$ \\
LR-TR equivocal & $7(17.5 \%)$ \\
LR-TR viable & $9(22.5 \%)$ \\
Total & 40 \\
\hline
\end{tabular}

Data in parentheses are percentages. Percentages may total $100 \%$ because of rounding. LI-RADS, Liver Imaging Reporting and Data System; TRA, tumor response algorithm.

In our study, patients classified as LR-TR viable showed significantly lower OS than those classified as LR-TR nonviable and LR-TR equivocal. However, there was no significant difference in OS between LR-TR nonviable and LR-TR equivocal. We speculate that one the reason for this result might be that the observers had a better grasp of the features of tumor viability, while there was no consensus on the expected imaging features posttreatment, including the pseudo-progressed lesions after treatment and the thin, smooth rind of enhancing peripheral liver tissue, which resulted in the inappropriate use of the tie-breaking rule in LI-RADS TRA. Alternatively, this might also be related to the period of posttreatment image acquisition in this study. As there is no relevant research to guide the selection of the time interval of the posttreatment images for tumor response evaluation by LI-RADS TRA, the interval used in the present study might have been less than optimal in providing quality evidence for carefully defining LI-TR equivocal, and more research needs to be conducted in this regard.

Our study had several limitations worth noting. First, some selection bias existed due to the single-center, retrospective design. Second, different CT machines and scanning parameters might have influenced the interpretation of imaging features and thus affected the interobserver agreement. Third, there was no pathological gold standard to test the efficacy of LI-RADS TRA in this study. We also acknowledge that imaging is far less accurate than pathology in determining whether a tumor has complete necrosis; however, survival outcomes of all the patients were collected in our study, and thus, we think it would be better for evaluating the accuracy and utility of LI-RADS 2018 TRA. Also, the time of posttreatment image acquisition in this study was also a limitation. As the time interval of posttreatment is not clearly defined in LI-RADS 2018 TRA, it is important to find a proper time interval to validate the performance of LI-RADS 2018 TRA, and this may be a key avenue of research in the future. Furthermore, number of patients included in this study was relatively small, but this was, as far as we know, largely a byproduct of the strict inclusion and treatment criteria for the application of RFA in patients with HCC (27-29); accordingly, we developed a very strict patient screening process to better evaluate the efficacy of LI-RADS version 2018 CT TRA for treating HCC. Further prospective studies are needed to determine the efficiency of our results regarding the performance of the LI-RADS 2018 TRA in tumor response evaluation and survival prediction in patients with HCC after RFA. Moreover, it is also necessary to explore the efficiency of other imaging technologies and compare the performance of LI-RADS TRA with other evaluation criteria.

\section{Conclusions}

Overall, our study showed that LI-RADS version 2018 TRA can be used to predict viable or nonviable of HCC after RFA. Patients classified as LR-TR viable had significantly lower OS than LR-TR nonviable and LR-TR equivocal patients. However, the OS between LR-TR nonviable and LR-TR equivocal did not differ significantly. Further explorative and prospective studies are needed to validate the performance of the LI-RADS 2018 TRA in tumor response evaluation and survival prediction in patients with HCC after RFA and other locoregional treatment. The application of other imaging techniques and the 

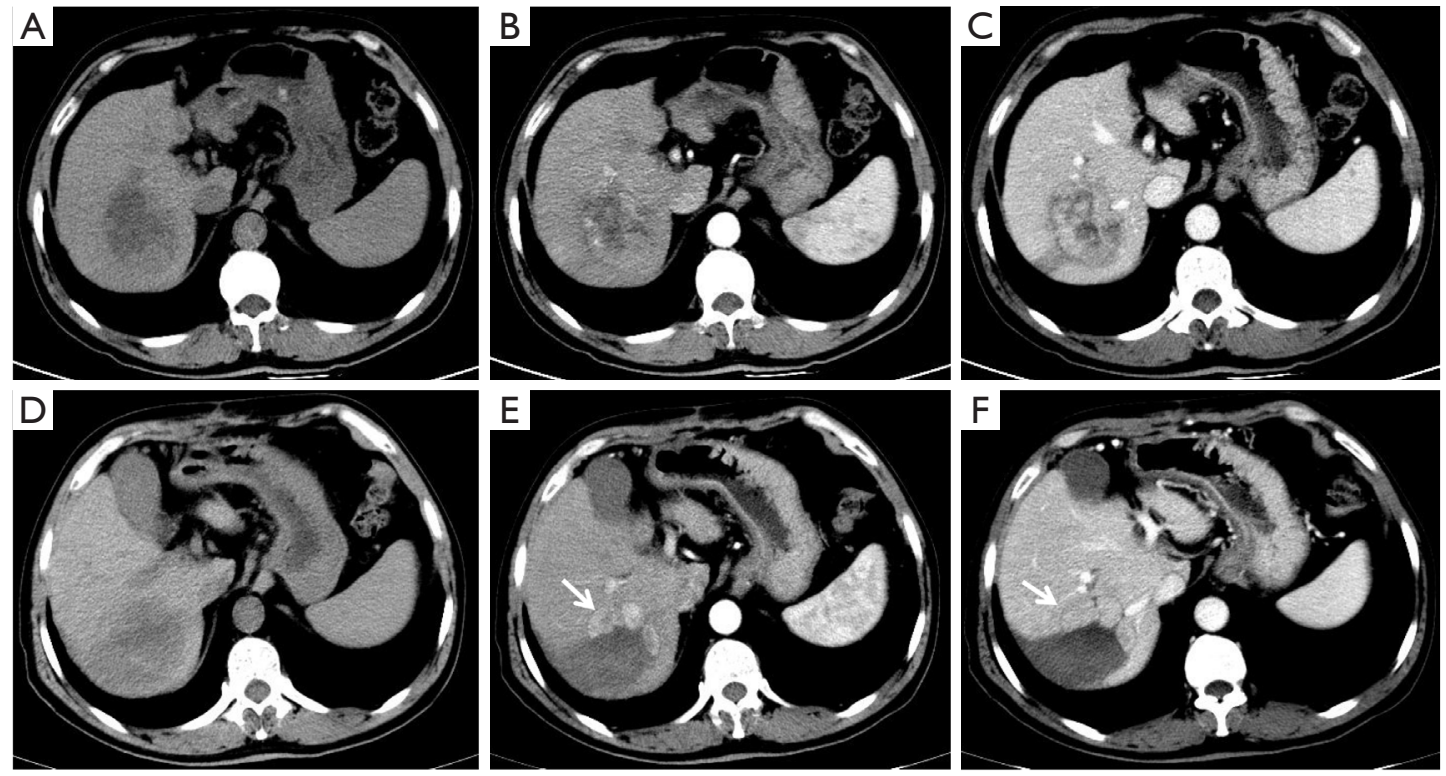

Figure 2 A 70-year-old man with hepatocellular carcinoma (HCC) in the VII segment of the liver. A tumor $6.9 \mathrm{~cm}$ in size in the right lobe of liver with nonuniform low-density (A), showing hyperenhancement in the arterial phase (B), and continuous enhancement without distinct "washout" appearance in the portal venous phase (C); after radiofrequency ablation (RFA), a non-enhanced ablation area was found in original lesion area, along with a $5.0 \mathrm{~cm}$-sized irregular, thickened tissue area (arrows) along the margin of the treated lesion (D), showing hyperenhancement in the arterial phase $(\mathrm{E})$ and washout $(\mathrm{F})$. Diagnosis was agreed upon by the 2 readers (LR-TR viable $5.0 \mathrm{~cm})$.
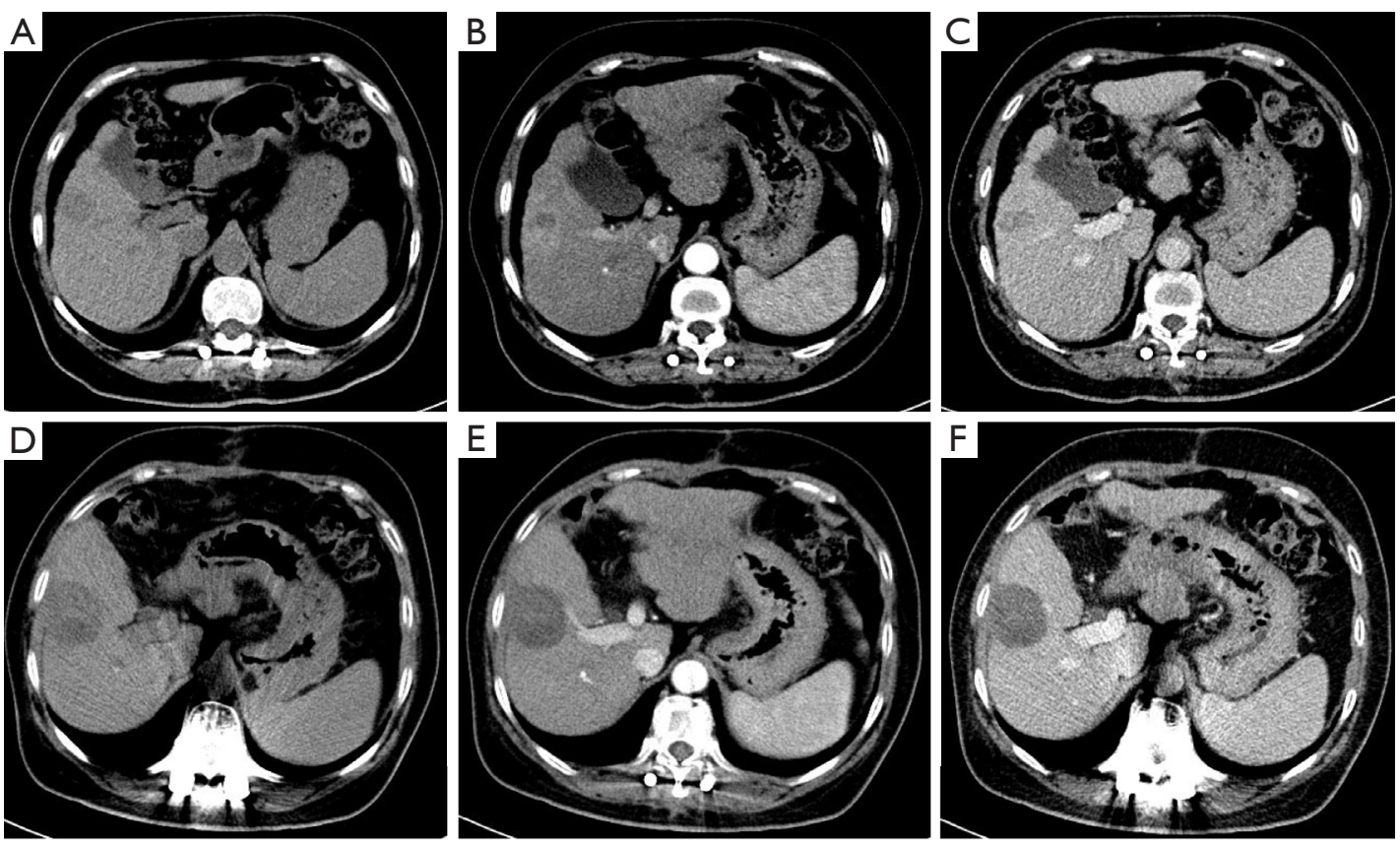

Figure 3 A 72-year-old woman with hepatocellular carcinoma (HCC) in the V segment of the liver. A low-density tumor $2.5 \mathrm{~cm}$ in size on normal abdominal CT images (A), showing hyperenhancement (rim) in the arterial phase (B), "washout" appearance, and enhanced capsule (arrow) in the portal venous phase (C); after radiofrequency ablation (RFA), a non-enhanced ablation area was shown (D), and there was not any enhancement tissue in or along the margin of the treated lesion (E,F). Diagnosis was agreed upon by the 2 readers (LR-TR nonviable). 

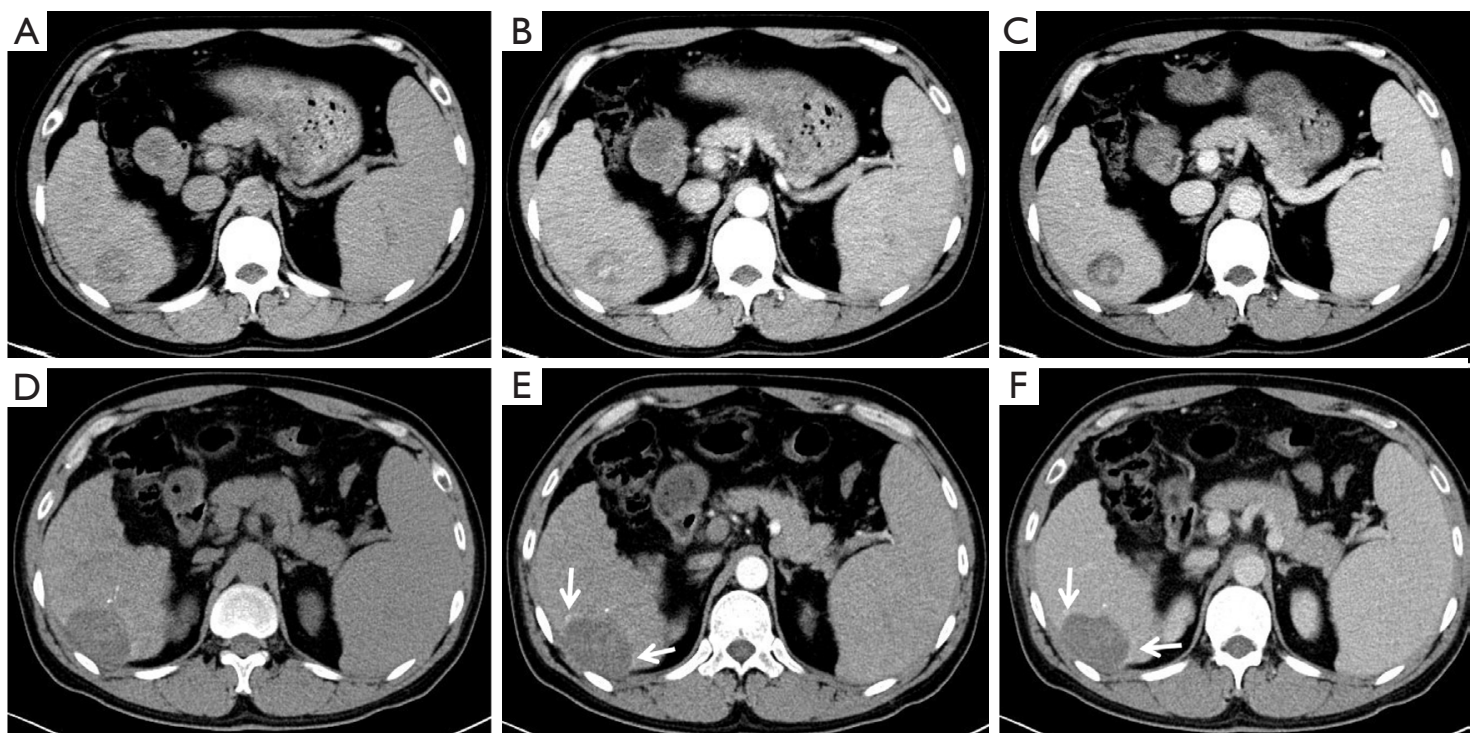

Figure 4 A 48-year-old man with hepatocellular carcinoma (HCC) in the VI segment of the liver. A low-density lesion in the right lobe of the liver $2.7 \mathrm{~cm}$ in size on normal abdominal CT images (A) with nonuniform hyperenhancement (not rim) in the arterial phase (B) and "washout" appearance in the portal venous phase (C); after radiofrequency ablation (RFA), a non-enhanced ablation area was shown (D), but with no nodular or irregular enhancement except for an nonsmoothed rim (arrows) of enhancement on arterial (E) and portal venous phases $(\mathrm{F})$. There was inconsistent diagnosis between the 2 readers (R1: equivocal; R2: nonviable. The final conclusion re-valuated by R3: equivocal).

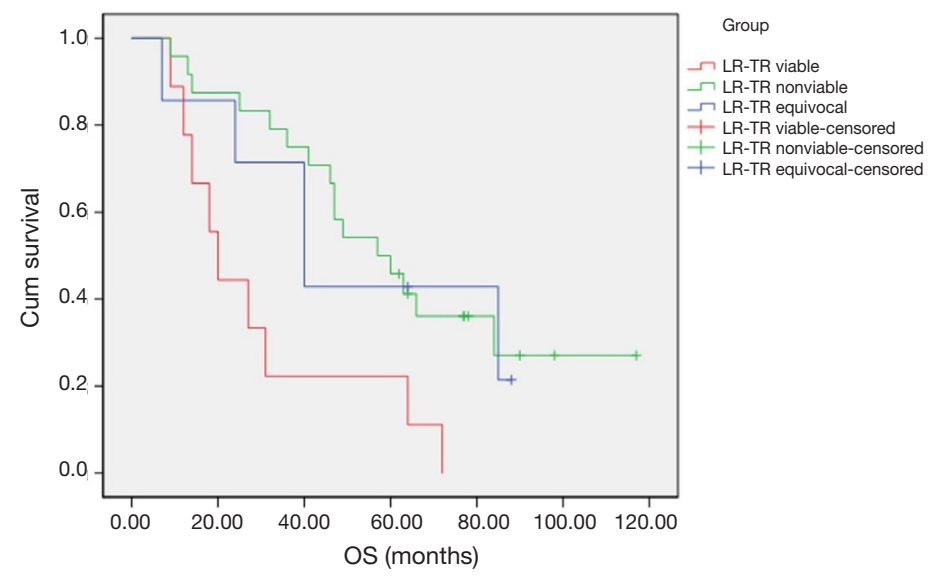

Figure 5 Survival curves among the three LR-TR catagories. Patients classified as LR-TR viable had significantly lower OS than those classified as LR-TR nonviable $(\mathrm{P}=0.005)$ and LR-TR equivocal $(\mathrm{P}=0.036)$. However, the OS between LR-TR nonviable and LR-TR equivocal did not differ significantly $(\mathrm{P}=0.901)$. LR-TR, Liver Imaging Reporting and Data System Tumor Response; OS, overll survival. 
comparative study with other evaluation criteria would also be highly valuable.

\section{Acknowledgments}

Funding: The National Natural Science Foundation of China (Grant number 81771797), The 1.3.5 Project for Disciplines of Excellence, West China Hospital, Sichuan University (Grant number ZYJC18008), and the National Nature Science Foundation of China (Grant number 81971571).

\section{Footnote}

Conflicts of interest: The authors have no conflicts of interest to declare.

Ethical Statement: The authors are accountable for all aspects of the work in ensuring that questions related to the accuracy or integrity of any part of the work are appropriately investigated and resolved. Institutional review board approval (ID: 2019-962) was obtained before this retrospective study was conducted.

Open Access Statement: This is an Open Access article distributed in accordance with the Creative Commons Attribution-NonCommercial-NoDerivs 4.0 International License (CC BY-NC-ND 4.0), which permits the noncommercial replication and distribution of the article with the strict proviso that no changes or edits are made and the original work is properly cited (including links to both the formal publication through the relevant DOI and the license). See: https://creativecommons.org/licenses/by-nc-nd/4.0/.

\section{References}

1. Bray F, Ferlay J, Soerjomataram I, et al. Global cancer statistics 2018: GLOBOCAN estimates of incidence and mortality worldwide for 36 cancers in 185 countries. CA Cancer J Clin 2018;68:394-424.

2. Narsinh KH, Duncan DP, Newton IG, et al. Liverdirected therapy for hepatocellular carcinoma. Abdominal radiology (New York) 2018;43:203-17.

3. Miller AB, Hoogstraten B, Staquet M, et al. Reporting results of cancer treatment. Cancer 1981;47:207-14.

4. Eisenhauer EA, Therasse P, Bogaerts J, et al. New response evaluation criteria in solid tumours: Revised RECIST guideline (version 1.1). Eur J Cancer 2009;45:228-47.
5. Therasse P, Arbuck SG, Eisenhauer EA, et al. New guidelines to evaluate the response to treatment in solid tumors. European Organization for Research and Treatment of Cancer, National Cancer Institute of the United States, National Cancer Institute of Canada. J Natl Cancer Inst 2000;92:205-16.

6. Bruix J, Sherman M, Llovet JM, et al. Clinical management of hepatocellular carcinoma. Conclusions of the Barcelona-2000 EASL conference. European Association for the Study of the Liver. J Hepatol 2001;35:421-30.

7. Choi H, Charnsangavej C, de Castro Faria S, et al. CT evaluation of the response of gastrointestinal stromal tumors after imatinib mesylate treatment: a quantitative analysis correlated with FDG PET findings. AJR Am J Roentgenol 2004;183:1619-28.

8. Lencioni R, Llovet JM. Modified RECIST (mRECIST) assessment for hepatocellular carcinoma. Semin Liver Dis 2010;30:52-60.

9. Shim JH, Lee HC, Kim SO, et al. Which response criteria best help predict survival of patients with hepatocellular carcinoma following chemoembolization? A validation study of old and new models. Radiology 2012;262:708-18.

10. Jung ES, Kim JH, Yoon EL, et al. Comparison of the methods for tumor response assessment in patients with hepatocellular carcinoma undergoing transarterial chemoembolization. J Hepatol 2013;58:1181-7.

11. Gordic S, Corcuera-Solano I, Stueck A, et al. Evaluation of HCC response to locoregional therapy: Validation of MRI-based response criteria versus explant pathology. J Hepatol 2017;67:1213-21.

12. Xie X, Jiang C, Peng Z, et al. Local Recurrence after Radiofrequency Ablation of Hepatocellular Carcinoma: Treatment Choice and Outcome. J Gastrointest Surg 2015;19:1466-75.

13. Mitchell DG, Bruix J, Sherman M, et al. LI-RADS (Liver Imaging Reporting and Data System): summary, discussion, and consensus of the LI-RADS Management Working Group and future directions. Hepatology 2015;61:1056-65.

14. Elsayes KM, Hooker JC, Agrons MM, et al. 2017 Version of LI-RADS for CT and MR Imaging: An Update. Radiographics 2017;37:1994-2017.

15. Marrero JA, Kulik LM, Sirlin CB, et al. Diagnosis, Staging, and Management of Hepatocellular Carcinoma: 2018 Practice Guidance by the American Association for the Study of Liver Diseases. Hepatology 2018;68:723-50.

16. Chaudhry M, McGinty KA, Mervak B, et al. The LIRADS Version 2018 MRI Treatment Response Algorithm: 
Evaluation of Ablated Hepatocellular Carcinoma. Radiology 2020;294:320-6.

17. Cools KS, Moon AM, Burke LMB, et al. Validation of the Liver Imaging Reporting and Data System Treatment Response Criteria After Thermal Ablation for Hepatocellular Carcinoma. Liver Transpl 2020;26:203-14.

18. Thibodeau-Antonacci A, Petitclerc L, Gilbert G, et al. Dynamic contrast-enhanced MRI to assess hepatocellular carcinoma response to Transarterial chemoembolization using LI-RADS criteria: A pilot study. Magn Reson Imaging 2019;62:78-86.

19. Shropshire EL, Chaudhry M. LI-RADS Treatment Response Algorithm: Performance and Diagnostic Accuracy. Radiology 2019;292:226-34.

20. Seo N, Kim MS, Park MS, et al. Evaluation of treatment response in hepatocellular carcinoma in the explanted liver with Liver Imaging Reporting and Data System version 2017. Eur Radiol 2020;30:261-71.

21. Zidan M, Thomas RL, Slovis TL. What you need to know about statistics, part II: reliability of diagnostic and screening tests. Pediatr Radiol 2015;45:317-28.

22. An C, Lee CH, Byun JH, et al. Intraindividual Comparison between Gadoxetate-Enhanced Magnetic Resonance Imaging and Dynamic Computed Tomography for Characterizing Focal Hepatic Lesions: A Multicenter, Multireader Study. Korean J Radiol 2019;20:1616-26.

23. Erkan B, Meier J, Clark TJ, et al. Non-invasive diagnostic

Cite this article as: Zhang Y, Wang J, Li H, Zheng T, Jiang H, Li M, Song B. Performance of LI-RADS version 2018 CT treatment response algorithm in tumor response evaluation and survival prediction of patients with single hepatocellular carcinoma after radiofrequency ablation. Ann Transl Med 2020;8(6):388. doi: 10.21037/atm.2020.03.120 criteria of hepatocellular carcinoma: Comparison of diagnostic accuracy of updated LI-RADS with clinical practice guidelines of OPTN-UNOS, AASLD, NCCN, EASL-EORTC, and KLSCG-NCC. PLoS One 2019;14:e0226291.

24. Ko A, Park HJ, Lee ES, et al. Comparison of the diagnostic performance of the 2017 and 2018 versions of LI-RADS for hepatocellular carcinoma on gadoxetic acid enhanced MRI. Clin Radiol 2020;75:319.e1-319.e9.

25. Basha MAA, Refaat R, Mohammad FF, et al. The utility of diffusion-weighted imaging in improving the sensitivity of LI-RADS classification of small hepatic observations suspected of malignancy. Abdom Radiol (NY) 2019;44:1773-84.

26. Lee S, Kim MJ, Kim SS, et al. Retrospective comparison of EASL 2018 and LI-RADS 2018 for the noninvasive diagnosis of hepatocellular carcinoma using magnetic resonance imaging. Hepatol Int 2020;14:70-9.

27. Livraghi T, Lazzaroni S, Meloni F. Radiofrequency thermal ablation of hepatocellular carcinoma. Eur J Ultrasound 2001;13:159-66.

28. Yan K, Chen MH, Yang W, et al. Radiofrequency ablation of hepatocellular carcinoma: long-term outcome and prognostic factors. Eur J Radiol 2008;67:336-47.

29. Eggert T, Greten TF. Current Standard and Future Perspectives in Non-Surgical Therapy for Hepatocellular Carcinoma. Digestion 2017;96:1-4. 